\title{
Ovarian endometriosis, a precursor of ovarian cancer: Histological aspects, gene expression and microRNA alterations (Review)
}

\author{
ADRIANA IOANA GAIA-OLTEAN ${ }^{1}$, CORNELIA BRAICU $^{2}$, DIANA GULEI $^{3}$, RAZVAN CIORTEA $^{4}$, \\ DAN MIHU ${ }^{4}$, HORACE ROMAN $^{5}$, ALEXANDRU IRIMIE $^{1,2}$ and IOANA BERINDAN-NEAGOE ${ }^{2,3,6}$
}

\author{
${ }^{1}$ Department of Oncological Surgery, Iuliu Hatieganu University of Medicine and Pharmacy, 400012 Cluj-Napoca; \\ ${ }^{2}$ Research Center for Functional Genomics and Translational Medicine; ${ }^{3}$ MedFuture-Research Center for Advanced Medicine, \\ Iuliu Hatieganu University of Medicine and Pharmacy, 40015 Cluj-Napoca; ${ }^{4}$ Second Department of Obstetrics and \\ Gynecology, Iuliu Hatieganu University of Medicine and Pharmacy, 400124 Cluj-Napoca, Romania; \\ ${ }^{5}$ Center of Endometriosis, Clinique Tivoli-Ducos, 33000 Bordeaux, France; ${ }^{6}$ Department of Functional Genomics and \\ Experimental Pathology, Oncology Institute 'Prof. Dr. Ion Chiricuta', 400015 Cluj-Napoca, Romania
}

Received March 7, 2020; Accepted December 17, 2020

DOI: $10.3892 / \mathrm{etm} .2021 .9674$

\begin{abstract}
Ovarian endometriosis is a frequent chronic gynecological disease with an uncertain evolution regarding its progression or association with ovarian malignant lesions. The present review summarized the histological aspects, gene expression and microRNA (miRNA/miR) alterations associated with ovarian endometriosis and cancer and their possible interaction. The endometriosis-ovarian cancer interaction has been proposed by certain researchers as a single entity. Histological results indicated that endometriosis has been in different circumstances coexisting with ovarian cancer, with reference to endometrioid and clear cell carcinoma. Endometriosis with moderate and severe atypia can influence cell proliferation and architecture, resulting in a possible malignant transformation. Gene expression analysis indicated that the pathologies of both endometriosis and ovarian cancer are characterized by genetic instability from a molecular point of view, as several important genetic mutations, including ARID1A, PI3KCA, PTEN, BRCA1, BRCA2, TP53 and KRAS genes, were identified. miRNA alterations have been implicated in the regulation of gene expression. Common dysregulated miRNAs, such as miR-331, miR-335, miR-891,
\end{abstract}

Correspondence to: Professor Ioana Berindan-Neagoe, Research Center for Functional Genomics and Translational Medicine, Iuliu Hatieganu University of Medicine and Pharmacy, 23 Gheorghe Marinescu Street, 40015 Cluj-Napoca, Romania

E-mail: ioananeagoe29@gmail.com

Abbreviations: EAOC, endometriosis-associated ovarian cancer; miRNAs, microRNAs

Key words: ovarian endometriosis, ovarian cancer, histology, gene expression, miRNAs
miR-548, miR-124, miR-148, miR-215, miR-192, miR-337, miR-153, miR-155, miR-144, miR-221 and miR-3688 were extensively investigated in understanding endometriosis and ovarian cancer evolution. From a combined viewpoint including histological aspects, gene expression and miRNA alterations, it is reasonable to speculate that endometriosis is associated with ovarian cancer. Ovarian endometriosis lesions may present a risk for ovarian malignant lesions, which supports a model of endometriosis as a malignant precursor. However, the endometriosis-ovarian cancer association is not widely accepted in the literature and additional studies are required to validate this association.

\section{Contents}

1. Introduction

2. Histological aspects

3. Gene expression

4. miRNA alterations

5. Impact of endometriosis on the prognosis of ovarian cancer

6. Conclusion

\section{Introduction}

Endometriosis is one of the most painful and frequent chronic gynecological diseases that is characterized by the presence and growth of endometrial ectopic tissue outside the uterus (1). The incidence and prevalence of endometriosis are difficult to quantify, considering that some patients with this pathology are asymptomatic. Hsiao et al (2) mentioned a prevalence rate of 10-15\% among all women of reproductive age worldwide. In particular, ovarian endometriosis is one of the most frequent subtype that appears in 17-44\% women diagnosed with endometriosis (3).

Sampson (4) in 1925, was the first to present with histological details the complex mechanism of the malignant transformation 
of endometriosis and its association with epithelial ovarian cancer. Subsequently, Jiang et al (5) indicated that progression of endometriosis to ovarian cancer was corroborated by molecular data. At present, the two pathologies are frequently regarded as a single histological entity, which is known as endometriosis-associated ovarian cancer (EAOC) (6). The association between ovarian endometriosis and ovarian cancer is supported by epidemiological data debated in several studies (1,5-14).

Ovarian cancer represents a complex and heterogeneous malignant gynecological pathology (15) with a high mortality rate, with and a 5 -year survival rate $<45 \%$ (16), which is considered to be the second most common gynecologic malignancy in developed countries (17). The risk of ovarian cancer development is $\sim 1 \%$ among patients during lifetime in developed countries (18).

In concordance with the histological modifications, the dynamics in coding and non-coding gene expression at cellular level is a decisive factor regarding the cellular phenotype (19). Among these transcripts, microRNAs (miRNAs/miRs), which are small sequences of 19-22 nucleotides in length without coding capacity, have been implicated in post-transcriptional regulation of protein expression (19). miRNAs are capable of regulating complex signaling networks due to their ability to target and influence the translation of coding genes (20). In the case of cancer, miRNAs are classified as tumor suppressors or oncogenes depending on their target genes and also their level of expression (21). The profile of differentially expressed miRNAs in patients with ovarian cancer and endometriosis was analyzed (22). The expression of miRNAs between ectopic and eutopic endometrium in patients with and without endometriosis offered information with diagnostic, prognostic or even therapeutic implications $(23,24)$.

The present review focused on emphasizing the main histological aspects, gene expression and miRNAs alterations in ovarian endometriosis and cancer and their possible association, based on the latest published literature.

\section{Histological aspects}

Histology represents the study of the arrangement for various tissues, which requires a microscopic knowledge of the microanatomy (25). Histopathology is a branch of histology that involves the microscopic identification and study of diseased tissue that not only establish the diagnosis but is also crucial for providing prognostic in data clinical management (26).

Ovarian endometriosis. In a histopathological perspective, endometriosis is defined as the presence of endometrial-like glandular epithelium and stroma outside the uterus. At the immunohistochemical level, the phenotype of endometrial glands is similar to that of normal endometrial tissue (27). However, there are certain microscopic alterations specific of ovarian endometriosis, which are listed in Table I and illustrated in Fig. 1.

Regarding the presence of ectopic endometrial epithelium, several studies have documented the presence of hyperplasia and/or atypia in cytologic endometrial lesions (25-27). Atypical endometriosis has been indicated to confer a higher increase risk of malignant transformation to ovarian cancer (28). A total of $\sim 8 \%$ of ovarian endometriosis exhibits histopathological features of atypical endometriosis (4). The presence of hyperplastic lesions in the glandular epithelium is less frequent compared with that in atypia but may be present in some ovarian endometriosis cysts (28).

Ovarian cancer. Ovarian cancer is classified in two distinct subgroups, type I and II, taking into account the clinical features, histopathological characteristics and gene expression pattern $(24,27)$. Type I subgroup is characterized by a low rate of proliferation, while type II subgroup exhibits a higher proliferation rate and is more aggressive $(24,27)$. In addition, type I subgroup includes low-grade serous, borderline serous, mucinous, endometrioid and clear cell carcinoma, while type II subgroup is comprises high-grade serous carcinoma, mixed malignant mesodermal carcinosarcomas and undifferentiated carcinoma $(28,29)$.

Two subcategories of type I epithelial ovarian cancer, endometrioid and clear cell carcinoma, appear to be more often derived from or coexist with ovarian endometriosis $(9,10,18,30)$. These two subcategories are the most frequent types after serous carcinoma. Two previous population-based studies which reviewed the pathology using modern diagnostic criteria presented an estimated frequency of $12-13 \%$ for clear cell and $9-11 \%$ for endometrioid carcinoma, among $20 \%$ of all epithelial ovarian cancer $(18,31)$.

The main histological characteristics of these two ovarian cancer subtypes are presented in Table II and Fig. 2.

$E A O C$. EAOC is defined as one of the following three conditions: i) Detection in the same ovary of endometriosis and ovarian cancer; ii) detection of endometriosis in one ovary and of ovarian cancer in the other; iii) coinciding identification of ovarian cancer in any of the ovaries and pelvic endometriosis (28). Considering the initial assumption of Sampson regarding the malignant transformation of endometriosis to ovarian cancer (2), when currently EAOC is considered as a single entity, it has been defined as cumulative histological features characteristic to benign endometriosis, endometriosis contiguous with or associated with an ovarian malignancy and malignant lesions intercalated with several intermediary lesions (Fig. 3) (32).

Several studies reporting histopathological characteristics of EAOC have been performed. Of these, Fukunaga et al (33) reported atypical endometriosis for $54 \%$ of clear cell and $42 \%$ of endometrioid carcinoma. Ballouk et al (34) indicated that half of the endometrial cysts demonstrated severe atypia and presented with an invasive capacity of malignancies.

\section{Gene expression}

Abnormal cell transformations, such as endometriosis and ovarian cancer, are sustained at molecular level via alterations in homeostatic gene expression profiles and signaling pathways (22). These changes often result in pathologically expressed proteins that finally lead to the alteration of cellular processes $(5,32)$.

Ovarian endometriosis. From a molecular point of view, ovarian endometriosis is characterized by a broad and 
Table I. Microscopic criteria for the diagnosis of endometriosis (27).

Type

Criteria

Endometrial type glandular epithelium and stroma with associated constellation of findings

Endometrioid cylindrical epithelium
Granulation tissue (macrophages)

Hemosiderin deposit

Fibrosis

Pseudoxanthoma cells

Island of residual glandular epithelium or endometrial stroma Hyperchromatic nucleus

Smudgy chromatin

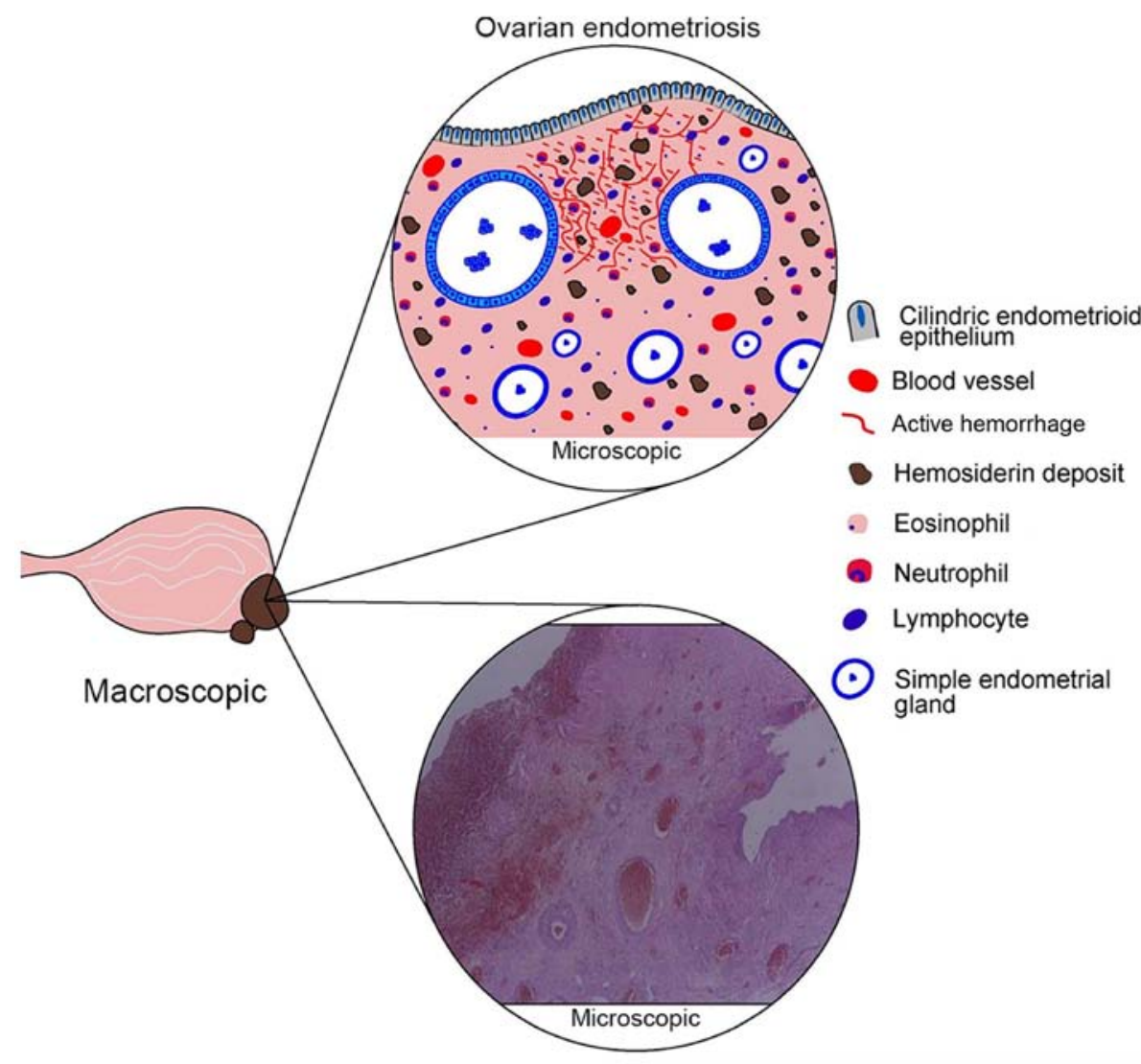

Figure 1. Histological aspects of ovarian endometriosis. In terms of macroscopic features, ovarian endometriosis usually takes the form of a cyst, well defined, unilocular or multilocular, varying in size, with chocolate cysts. In terms of microscopic features, schematic representation (upper image) with subsequent identification of important characteristics in the lower microscopic image outlines the defining endometriosis elements, which include cylindrical endometrial epithelium, endometrial-type glands (simple and/or sometimes atypical), endometrial stroma containing hemosiderin deposits, active bleeding, blood vessels and inflammatory infiltrates (eosinophils, neutrophils and lymphocytes).

important genetic variety which can result in a wide genetic instability (32). Histologically, molecular abnormalities can be concealed in benign endometriosis, which subsequently may lead to a malignant transformation (28).

In ovarian endometriosis, several studies have reported genetic mutations with important contributions in this pathology and its possible malignant transformation (Table III).

ARID1A is a gene identified to exhibit a tumor suppressor role, and the loss of ARID1A expression is considered to be responsible for the activation of early carcinogenic mechanisms (28). Mutations of ARID1A have been indicated to be directly connected with atypical endometriosis (35). However, no alterations in the ARID1A expression level have been identified in paired samples of distal nonatypical endometriotic tissue (27).

PTEN, which is another tumor suppressor gene, has been reported to be present in endometriotic lesions, while it has been indicated that PTEN inactivation exhibits a significant role in the malignant evolution of endometriosis (36).

PIK3CA has been recognized for its oncogenic role. Mutations in this gene have been evaluated in nonatypical and atypical endometriosis, and are considered to be an early 
Table II. Microscopic criteria for the diagnosis of endometrioid and clear cell ovarian cancer (27).
Endometrioid ovarian carcinoma
Clear cell ovarian carcinoma

Glandular, cribriform or solid architecture and morular or squamous differentiation

Cuboid to columnar cells with grading similar to the uterine counterpart

Nuclear grade determined by the variation in nuclear size, chromatin distribution and size of the nucleoli

Mitoses present

Endometriosis often present
Tubulocystic, papillary or solid architecturea

Hobnail or polyhedral cells with abundant eosinophilic granular/clear cytoplasm, signet/ring-type cells

Round/angular, hyperchromatic nuclei, marked nuclear pleomorphism

Mitoses present

Endometriosis often present

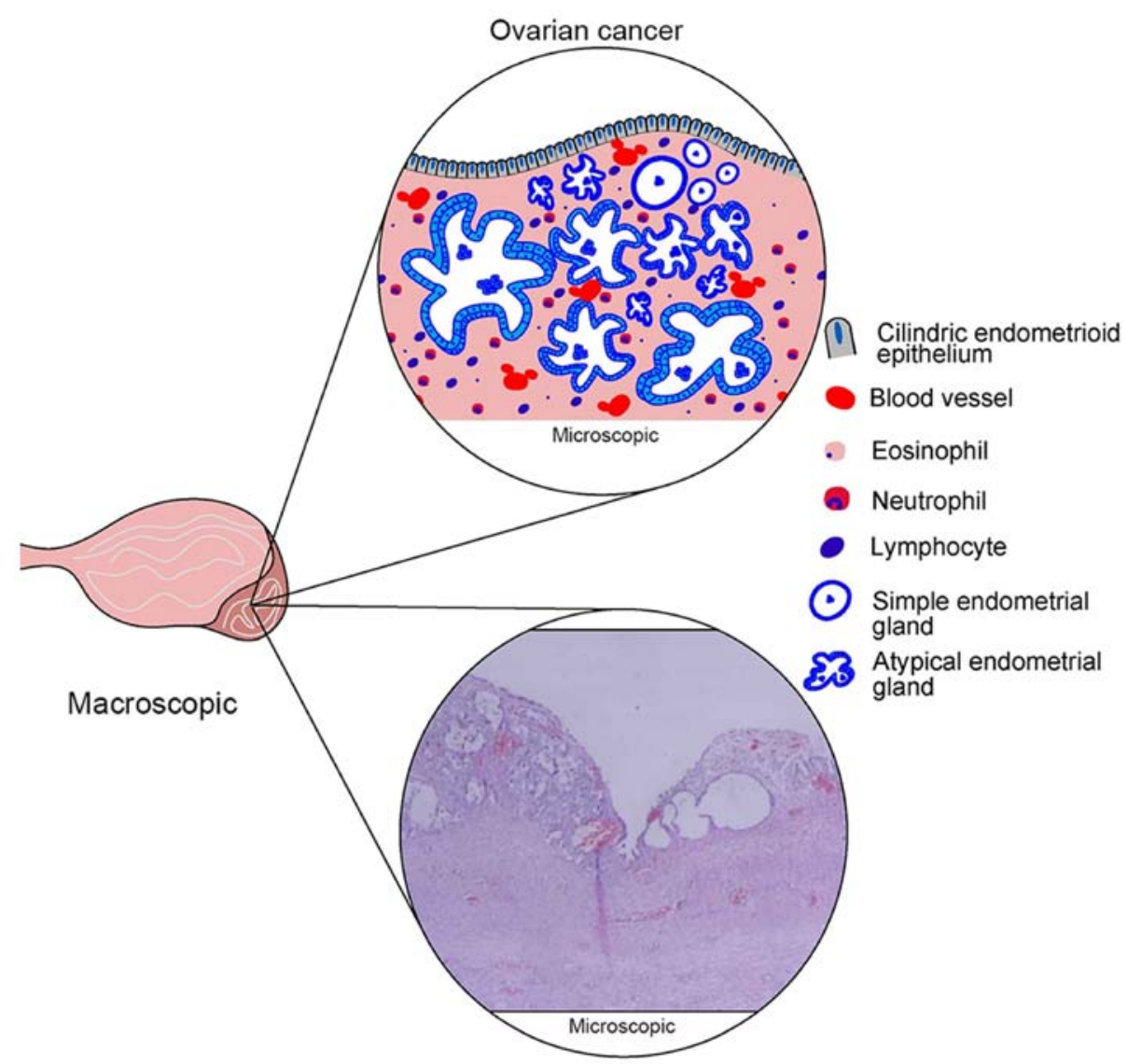

Figure 2. Histological aspects of ovarian cancer. In terms of macroscopic features, epithelial ovarian cancer (here referring to endometrioid and clear cell carcinoma) is a cumulative entity, presenting various forms with imprecise delimitation and variable dimensions. In terms of microscopic features, schematic representation (upper image) with subsequent identification of important characteristics in the lower microscopic image outlines the disordered architecture with numerous atypical glands and nuclear variations. These are associated with elements presented and described in the benign inflammatory endometriosis or the non-endometriotic process.

event in carcinogenesis, possibly at the beginning of malignant transformation of endometriosis (37).

CTNNB1 gene also exhibits an oncogenic function, which has been highlighted by its important role in the diagnosis of nonatypical and atypical endometriosis (38). CTNNB1 has been indicated to exhibit a prominent function in the early events of the transformation of endometriosis to ovarian cancer (38).

BRCA1 and BRCA2 are important early onset tumor suppressors genes. Mutations in the BRCA1 and BRCA2 genes have been reported in the evolution of various human cancers, including ovarian tumors. However, regarding their role in endometriosis, there are fewer reports $(18,39)$.

The presence of TP53 tumor suppressor gene mutations in atypical endometriosis is debated. Certain studies have indicated that alterations in the TP53 gene were present in atypical and low levels or absent in nonatypical endometriosis (37-39). It has been speculated, according to microarray results, that TP53 cancer-related pathways may participate in endometriosis progression (40).

KRAS is an oncogene, whose activation was detected in de novo endometriosis in mice. This suggested that activation 


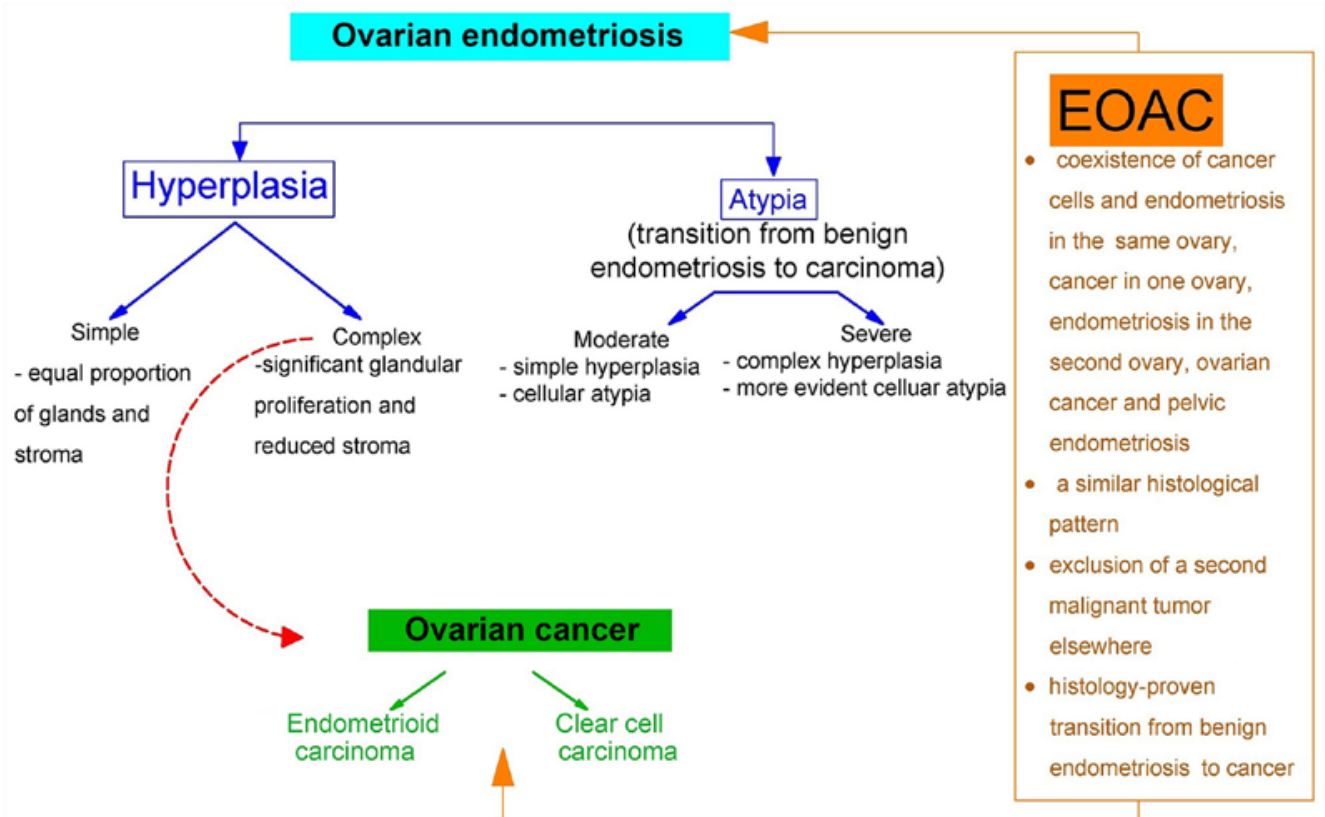

Figure 3. Overview of histological associations between ovarian endometriosis and the presence of malignant lesions. Ovarian endometriosis comprises different cellular alterations, such as simple/complex hyperplasia and moderate/severe atypia. These are present at certain moments of the evolution of endometrial lesions may be associated with ovarian cancer, such as endometrioid or clear cell carcinoma. The main histological characteristics of EAOC and their association with particular extrauterine sites are summarized. EAOC, endometriosis-associated ovarian cancer.

of KRAS is an important pathway in the initiation and progression of this disease (18). KRAS mutations have also been observed at an important level in the eutopic endometrium of patients with endometriosis (40).

Ovarian cancer. The classification of ovarian cancer based on histopathological and clinical features is accompanied by the gene expression pattern and mutational determination, which reflect the main factors responsible for the malignant transformation of ovarian cancer. These genes include KRAS, BRAF, PTEN, PIK3CA, CTNNB1 (the gene encoding $\beta$-catenin), ARID1A, PPP2R1A and rarely TP53 $(18,28,29,41)$. Considering the differentiation of ovarian cancers in two subtypes (I and II) as artificial and limiting in managing the complex biology of the disease, another classification consisting of five different subcategories has been proposed, based on clinical, morphological and molecular abnormalities (28).

Among all subcategories of ovarian cancer introduced in routine classification, the two subcategories associated with endometriosis, endometrioid and clear cell carcinoma, are extensively studied at the molecular level (42). In these two subcategories, gene mutation patterns have been identified in a different frequency, which conferred heterogeneous reports on the progression of malignant ovarian lesions (Table IV). According to the experimental data, the inactivation of ARID1A and PTEN tumor suppressor gene pathways and the activation of the oncogenic pathways regulated by PI3KCA, KRAS and CTNNB1 are responsible for initiating complex processes, which have been suggested as potential mechanisms associated with the early carcinogenesis and transformation of ovarian cancer $(28,38)$. Regarding the inactivating mutations in BRCA $1 / 2$ in endometrioid and clear cell carcinoma, there is no unanimously accepted opinion that BRCA gene mutations contribute to the pathology of the two cancers $(29,43)$. BRCA mutations or inactivation of gene expression have been indicated to occur more frequently in high grade serous carcinoma (29).

$E A O C$. The molecular pathways associated with the malignant transformation of EAOC remain to be fully elucidated. Endometriosis has been indicated to comprise mutations highlighted in coexisting tumors, which may designate a primary stage of evolution towards ovarian malignant transformation (44).

EAOC has been reported to exhibit a high percentage of PIK3CA and KRAS activating mutations and ARID1A and PTEN inactivating mutations $(35,37,38)$, whilst a reduced percentage of cases has been indicated to comprise TP53 and BRCA1/2 mutations $(45,46)$. A previous study has identified KRAS mutations in $29 \%$ of EAOC cases and only in $3 \%$ of tumors with no endometriosis (47). Lakhani et al (43) reported a smaller, but significant association between BRCA1/2 and endometrioid and clear cell carcinoma, namely for endometrioid: $33 \%$ of BRCA1, $29 \%$ of BRCA2 and for clear cell: $10 \%$ of BRCA; $1.4 \%$ of BRCA2.

\section{4. miRNA alterations}

Dysregulated miRNAs have been associated with a large number of human diseases, including cancer (48), cardiovascular disease (49), neurodegenerative disease $(50,51)$ and diabetes mellitus (52). They comprise information identified as molecular signatures, which are represented by specific panels of upregulated and downregulated molecules. The altered molecules define different profiles of gene or protein expression in various diseases (53). The differential transcriptional profiles 
Table III. Early abnormal molecular events in ovarian endometriosis.

\begin{tabular}{llc}
\hline Gene name & \multicolumn{1}{c}{ Function } & (Refs.) \\
\hline ARID1A & $\begin{array}{l}\text { Tumor suppressor gene } \\
\text { inactivation } \\
\text { Tumor suppressor gene }\end{array}$ \\
PTEN & $\begin{array}{l}\text { inactivation } \\
\text { Oncogene activation } \\
\text { PIK3CA }\end{array}$ & $\begin{array}{l}\text { Tumor suppressor gene } \\
\text { inactivation } \\
\text { BRCA1/BRCA2 }\end{array}$ \\
CTNNB1 & Oncogene activation \\
\hline
\end{tabular}

Table IV. Frequency of genetic alterations in endometrioid and clear cell ovarian carcinoma.

A, Endometrioid ovarian carcinoma

\begin{tabular}{lcc}
\hline Gene name & Frequency $(\%)$ & $($ Refs. \\
\hline ARID1A & 30 & $(81)$ \\
PTEN & 20 & $(29)$ \\
PIK3CA & 31 & $(38)$ \\
TP53 & 30 & $(18)$ \\
BRCA1 & 33 & $(43)$ \\
BRCA2 & 29 & $(43)$ \\
KRAS & 29 & $(47)$ \\
CTNNB1 & 40 & $(29)$ \\
\hline
\end{tabular}

$\mathrm{B}$, Clear cell ovarian carcinoma

\begin{tabular}{lcc}
\hline Gene name & Frequency $(\%)$ & $($ Refs.) \\
\hline ARID1A & 46 & $(81)$ \\
PTEN & 25 & $(44)$ \\
PIK3CA & 35 & $(38)$ \\
TP53 & 10 & $(18)$ \\
BRCA1 & 10 & $(43)$ \\
BRCA2 & 4 & $(43)$ \\
KRAS & 7 & $(82)$ \\
CTNNB1 & 3 & $(82)$ \\
\hline
\end{tabular}

of miRNAs between normal and pathological tissue samples may be used as diagnostic and prognostic tools (24). The interaction of miRNAs with different genes and their expression profiles is commonly specific for certain cell types and different stages of each disease (54). The present chapter presents the miRNAs that have been directly associated with the gene expression patterns previously reviewed in endometriosis and ovarian cancer and reported to be dysregulated in the two pathologies (Fig. 4).

Ovarian endometriosis. Endometriosis has been regarded as an ideal target for genomic sciences, since it lacks an efficient diagnostic and therapeutic management, as it represents a heterogeneous disease with multiple phenotypes and a complex pathophysiology (55). Several studies have indicated that miRNAs were implicated in the progression of endometriosis $(2,20,56)$. However, at present there is no specific clinical biomarker to be used in patients with endometriosis.

miR-200 family is one of the most widely studied miRNA families in endometriosis, and comprises miR-200a, miR-200b, miR-200c, miR-141 and miR-429 (57). miR-200b has been demonstrated to be the most downregulated transcript in previous studies on the molecular regulation of endometriosis (20). miRNAs of this family have been indicated to target PTEN and CTNNB1 genes (22).

miR-20a and miR-20b have been found to be dysregulated in ovarian endometriosis $(55,58)$. miR-20b has been indicated to contributes to the process of neovascularization in endometriosis (55) and target PTEN and BRCA1 genes.

miR-17-5p was reported by Jia et al (59) to be upregulated in patients with endometriosis compared with those without the disease, and has been indicated to target the PIK3CA gene.

Other miRNAs associated with endometriosis include $\mathrm{miR}-34 \mathrm{a} / \mathrm{b} / \mathrm{c}$, which were demonstrated by Burney et al (23) to be downregulated in ovarian endometriosis and target CTNNB1 and PTEN genes. miR-1-3p has been also reported to be upregulated in patients with endometriosis (20), and target the PIK3CA gene. The CTNNB1 gene has been indicated to be a target of miR-155-5p, which has been demonstrated to be dysregulated in endometriosis (60). miR-21-5p has been revealed to be involved in the pathogenesis of endometriosis exhibiting aberrant expression profiles (60) has been indicated to directly target BRCA1 and PTEN affecting their expression. PIK3CA, BRCA1 and BRCA2 have also been indicated to be targets of miR-335 (22).

Ovarian cancer. miRNAs present regulatory functions, certain of which exhibit important implications in carcinogenesis (61). Transcriptional microarray data have demonstrated that there is a different expression level of miRNAs between normal and tumor tissue (60). The up- and downregulation of miRNA expression have been associated with cancer development and progression (62). Alterations in miRNAs expression have been implicated in invasion and migration in ovarian carcinogenesis (63).

miR-200 family has been indicated to be involved in the metastasis of ovarian cancer (19). Notch signaling is considered to be a regulator of cell invasion in tumors (59). Notch signaling blockade via the miR-200 family has been indicated to represent a promising therapeutic approach for ovarian cancer (64).

miR-335 has been demonstrated to be downregulated in primary ovarian cancer tissue compared with normal tissue (65). Cao et al (65) also reported that patients with primary ovarian cancer who exhibited a low expression of miR-335 had a shorter survival period. In addition, miR-335 level has been revealed to be an important prediction factor of tumor recurrence (65). miR-335 has been indicated to target PIK3CA, BRCA1 and BRCA2 genes (22).

miR-17-5p has been reported to be overexpressed in ovarian cancer. Liu et al (66) demonstrated that miR-17 stimulated 


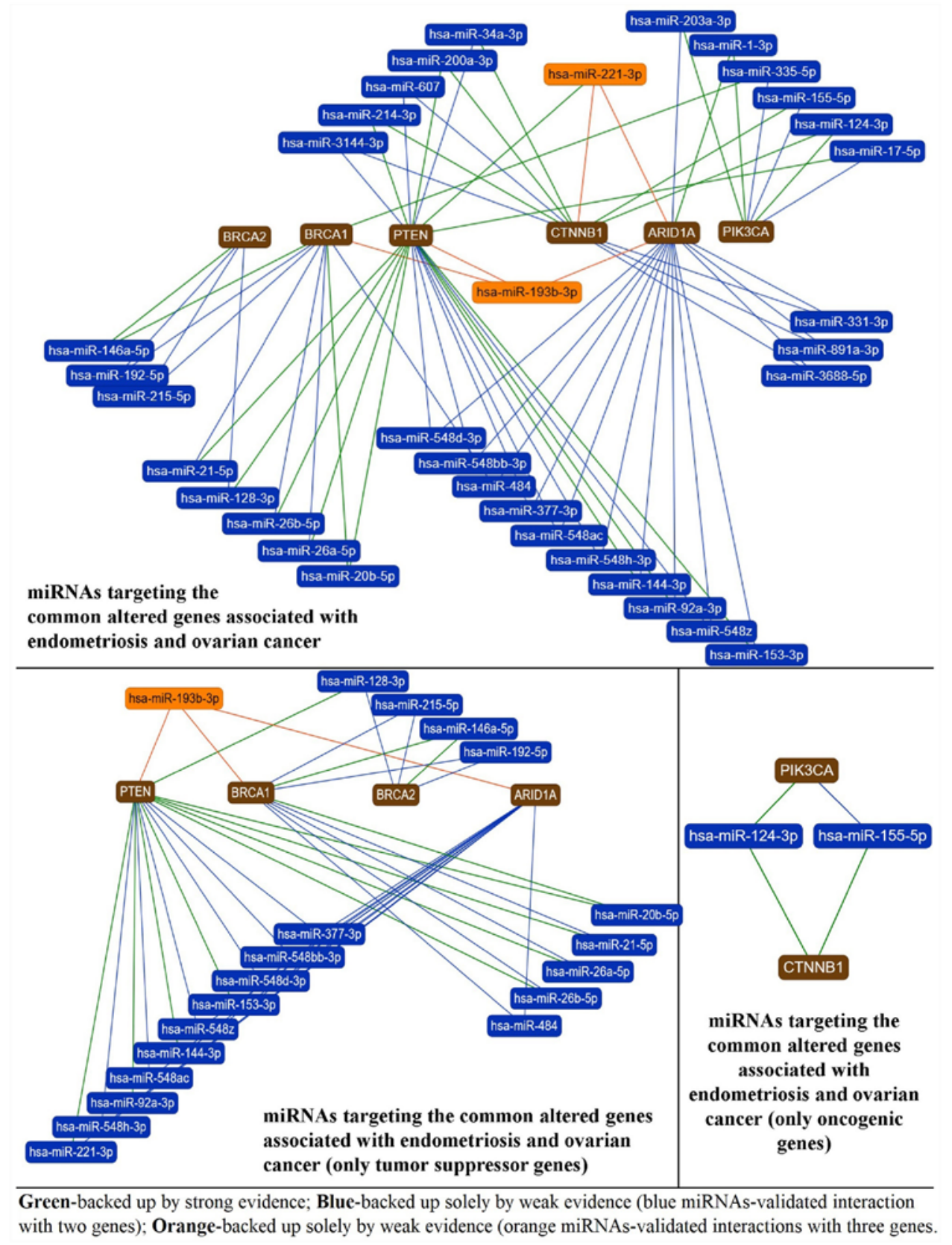

Figure 4. miRNAs targeting common altered genes in endometriosis and ovarian cancer. BRCA1, BRCA2, PTEN, CTNNB1, ARID1A and PIK3CA are altered in terms of mutation or expression in both endometriosis and ovarian cancer and are targeted by multiple miRNAs. The first graphical representation highlights miRNAs that have $>1$ of these genes in their target profile (miRNAs linked to the gene via green, blue or orange lines), with a special focus on miR-221-3p and miR-193b-3p that target simultaneously PTEN, CTNNB1, ARID1A (miR-221-3p) and BRCA1, PTEN and ARID1A (miR-193b-3p). The remaining miRNAs target simultaneously only two genes. The second graph illustrates the miRNAs that have $>1$ of these genes in their target profile, but only illustrates the tumor suppressor genes that are common between endometriosis and ovarian cancer (PTEN, BRCA1, BRCA2 and ARID1A). miR-193b-3p targets concomitantly three tumor suppressor genes and may become an important prognostic marker or therapeutic target in endometriosis and ovarian cancer or a marker of endometriosis transition toward ovarian malignancy, which requires validation. The third graph highlights the miRNAs that target simultaneously the two oncogenic genes (PIK3CA and CTNNB1) associated with endometriosis and ovarian cancer, miR-124-3p and miR-155-5p. The data were generated with miRTargetLink online software (22). miRNA/miR, microRNA.

the proliferation rate, accelerated cell cycle progression and affected the invasion capacity of the ovarian cancer cells in vitro. miR-17 has been also indicated to be involved in the transition from low to high degree ovarian cancer (66) and directly target the PIK3CA gene (22).

miR-34a-3p has been revealed to be frequently downregulated in ovarian cancer, being directly transactivated by the TP53 tumor suppressor gene, which is frequently dysregulated in ovarian epithelial cancer (67). miR-34 has been indicated to affect the motility, proliferation and migration of ovarian cancer cells (67) and directly target the CTNNB1 gene (22).

miR-1 level has been demonstrated to be decreased in ovarian cancer compared with normal ovarian tissue, indicating the potential tumor suppressor role of this gene (68).

miR-155-5p was revealed by Gulei et al (69) to exhibit increased expression in endometriosis, which was further accentuated in ovarian cancer samples but without statistical 
significance. miR-155 has been indicated to affect apoptosis and target the CTNNB1 gene (22).

miR-21-5p upregulation has been associated with ovarian cancer, where Liu et al (70) revealed that suppression of miR-21 reduced cell proliferation and promoted cell apoptosis by increasing PTEN expression. Apart from PTEN, miR-21 has been indicated to target the BRCA1 gene (22).

miR-148a is part of the miR-148/152 family.Downregulation of miR148/152 family members has been associated with unfavorable prognostic outcomes in ovarian cancer (71). miR-148a-5p targets the BRCA1 and BRCA2 genes (22).

Another miRNA associated with ovarian cancer is miR-20 which has been indicated to target the PIK3CA gene (22).

$E A O C$. Endometriosis presents a biological behavior with increased invasiveness similar to that of tumors. The invasion process is mediated by the downregulation of E-cadherin and alterations in the cell phenotype following epithelial-to-mesenchymal transition (72). This comprises a complex process converting the immotile epithelial cells into motile ones, as a response to injury (73). Therefore, endometriosis may be induced by epithelial-to-mesenchymal transition.

Fig. 4 presents the network of miRNAs-target genes in the context of ovarian endometriosis, EAOC and ovarian cancer. The three pathological states depicted in Fig. 4 have different common denominators of altered genes and miRNAs. However, large patient cohorts need to be employed before establishment of specific clinical signatures, such as a miRNA signature for the prediction of the evolution of endometriosis towards malignancy.

A synthesis of histological and molecular aspects encountered in ovarian endometriosis and ovarian cancer are highlighted in Fig. 5.

\section{Impact of endometriosis on the prognosis of ovarian cancer}

Endometriosis is considered by some specialists in the field as a pathology, although proof for its implications in ovarian cancer remain absent $(18,74)$; however, there are studies that demonstrate the contrary.

Braicu et al (19) performed a meta-analysis demonstrating that patients with endometriosis presented an increased risk of developing ovarian cancer. The data indicated an increased ovarian cancer risk by $27 \%$ in case-control or two-cohort studies, which included 314,421 females with or without endometriosis, and by $80 \%$ in single-arm cohort studies, which included 79,388 females with endometriosis (30). The results are in accordance with those of previous studies $(8,11)$ and they constitute epidemiological data that endometriosis may be associated with an increased risk of ovarian cancer (30).

Barreta et al (75) performed a database analysis between 1995 and 2016, aiming to clarify whether the clinicopathologies features and prognosis of patients with clear cell carcinoma and endometrioid carcinoma were associated with endometriosis. According to the original pathology report, 29 cases from a total of 55 cases, mentioned the presence of endometriosis. A second revision by an expert pathologist identified another 11 cases with endometrial foci. From the remaining 50 cases after exclusion criteria, 40 cases (80\%) were diagnosed as ovarian cancer associated with endometriosis (75).

Park et al (76) conducted a retrospective study between 1991 and 2012 that included 155 patients with clear cell carcinoma, 78 of which presented associated ovarian endometriosis.

Cases of EAOC in the aforementioned studies included young females with an early-stage disease, low-grade disease and specific histology of endometrioid and clear cell carcinoma. Ovarian endometriosis was strongly associated with an increased risk for ovarian cancer. In spite of favorable characteristics of EAOC, the findings indicated that endometriosis did not affect ovarian cancer prognosis after its onset, although a better overall survival was reported, primarily in clear cell carcinoma $(30,75,76)$.

\section{Conclusion}

Endometriosis, which is one of the most frequent gynecological diseases, requires a more comprehensive understanding. In addition, considering the possibilities of evolution of the endometrial lesions, a need for accuracy in diagnosis and a therapy plan is required (54).

The presence and increased number of cases of atypical endometriosis, considered by several studies as an intermediate lesion between endometriosis and ovarian malignancy, may allow the identification of endometrial lesions at risk for malignant transformation $(28,77,78)$. Since endometriosis is frequently observed in association with endometrioid and clear cell ovarian carcinoma, or in another perspective ovarian cancer arises from endometriosis, it can be hypothesized that endometriosis may be viewed as a preneoplastic or neoplastic process (36).

Moreover, the frequency of an aberrant mutation pattern seems to increase in cases of endometriosis adjacent or contiguous to ovarian cancer (28). Analyzing the presented aspects, it can be observed that all the gene mutations discussed (ARID1A, PI3KCA, PTEN, BRCA1/2, TP53 and KRAS) as being present in ovarian endometriosis $(18,28,36,39,40,79,80)$ are also present in endometrioid and clear cell carcinoma $(18,29,43,44,81,82)$, although certain among them (BRCA1/2, KRAS and TP53) are debated (39,40). Ovarian endometriosis and cancer tend to share the same genetic mutations, which also support the model of endometriosis as a malignant precursor. Development of genetic analyses to detect these mutations may represent a tool in the early detection of patients at risk to develop ovarian cancer (54).

miRNAs, which are involved in the regulation of gene expression, serve an important role in understanding endometriosis evolution and are currently extensively investigated (2). Increasing evidence suggests their role as biomarkers in endometriosis. miRNAs, such as miR-200, miR-17,miR-34,miR-1,miR-155 and miR-21, havebeen reported as being dysregulated in both endometriosis $(23,57,59,60)$ and ovarian cancer $(58,62,64-66,68)$, demonstrating the molecular association between these pathologies.

There are few studies that have indicated an association between ovarian endometriosis and ovarian cancer, but these data do exist (1). Ovarian endometriosis has been associated with an increased risk of ovarian cancer (36); however, no difference has been reported regarding prognosis in females with and without EAOC (18). 

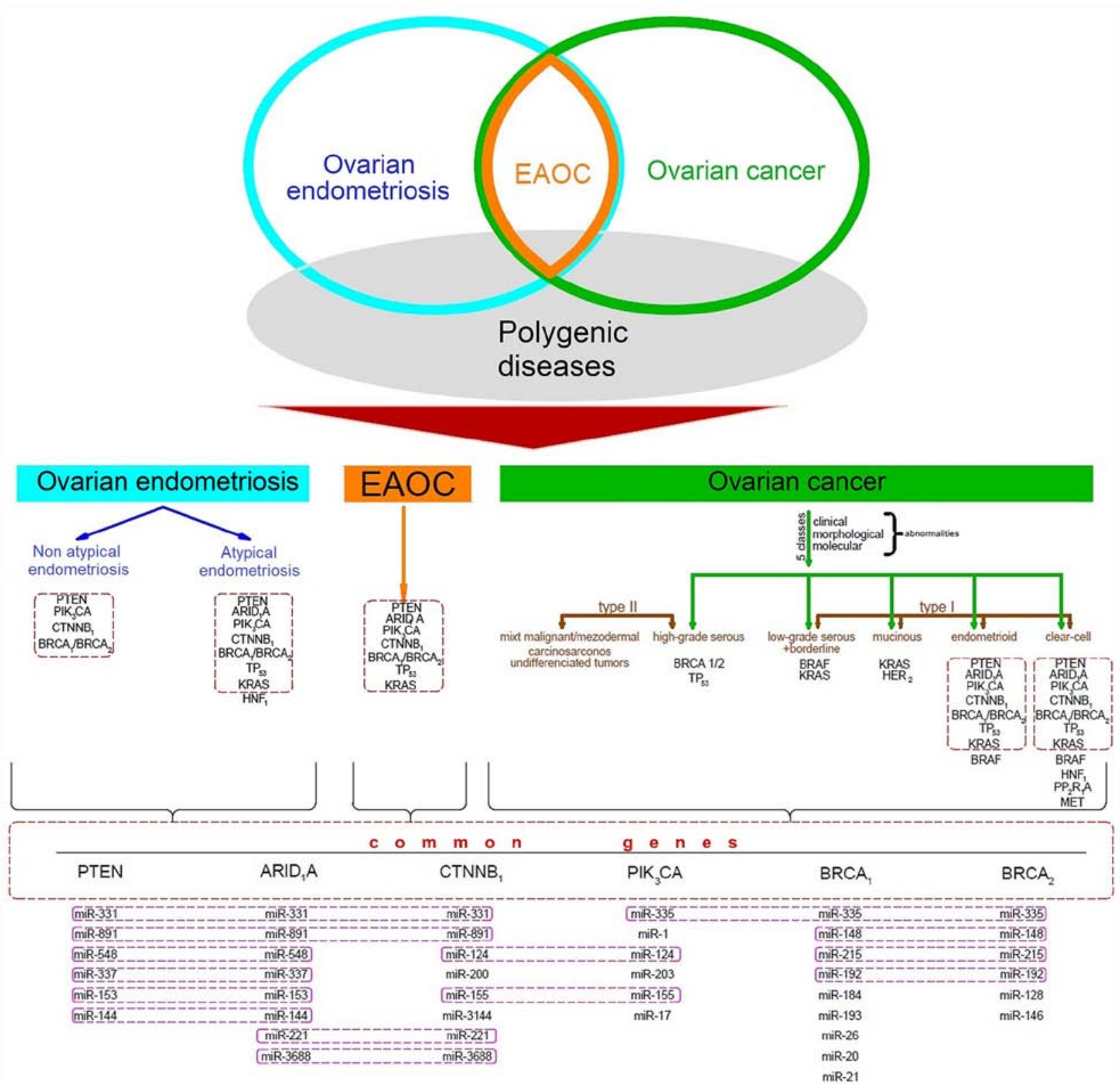

Figure 5. Overview of the histological and molecular association between ovarian endometriosis and ovarian malignant transformation. Molecular analysis of ovarian endometriosis focused on atypical and non-atypical endometriosis and ovarian cancer with focus on endometrioid ovarian carcinoma, clear cell ovarian carcinoma and EOAC lesions has identified the presence of certain genetic mutations and dysregulated miRNAs. Their comparison has demonstrated the presence of certain mutated genes in all three pathologies (highlighted with a red frame), which include PTEN, ARID1A, PIK3CA, CTNNB1, BRCA1/BRCA2, TP53, KRAS. These genes are targeted by certain commonly dysregulated miRNAs, such as miR-331, miR-335, miR-891, miR-548, miR-124, miR-148, miR-215, miR-192, miR-337, miR-153, miR-155, miR-144, miR-221, miR-3688 and other miRNAs dysregulated in both endometriosis and ovarian cancer, such as miR-200, miR-17, miR-34, miR-1, miR-155 and miR-21. EAOC, endometriosis-associated ovarian cancer; miRNA/miR, microRNA.

To elucidate the implications of the association of ovarian endometriosis and cancer, larger studies are required $(1,18)$. As a perspective, every group managing endometriosis and ovarian cancer should publish their data regarding this association. Further research on this topic with extensive validation is needed. Novel molecular technologies investigating epigenetic, transcriptomic, proteomic and post-translational splicing alterations and complex chromosomal rearrangements may revolutionize the management of endometriosis (2). The goal of these innovative efforts is the development of more sensitive diagnostic and preventive tests. miRNAs may represent an important tool in the management of endometriosis (21). Their potential use in diagnosis and treatment implications of endometriosis is a challenging and important step in its management. The early detection of the possible malignant lesions in females with endometriosis and the differentiation between women at risk of EAOC and those who will continue to present a benign disease is important in order to improve the preventive and diagnostic methods, as managing the situation after cancer has developed is not as efficient $(36,56)$. Until the materialization of these ideas, the caregivers in the clinics should pay attention in handling these cases.

\section{Acknowledgements}

Not applicable. 


\section{Funding}

The present review was funded by PhD research projects of the Iuliu Hatieganu University of Medicine and Pharmacy, Cluj-Napoca, Romania (grant nos. 7690/46/15.04.2016 and 5200/41/01.03.2017).

\section{Availability of data and materials}

Not applicable.

\section{Authors' contributions}

AIGO conceived the study, performed the histological, molecular and miRNA literature search and edited the manuscript. $\mathrm{CB}$ and DG performed the molecular and miRNA literature search and edited the manuscript. RC, DM and HR performed the clinical and histological literature search for endometriosis. AI performed the clinical and histological literature search for ovarian cancer. IBN supervised and critically revised the study. All authors read and approved the final manuscript.

\section{Ethics approval and consent to participate}

Not applicable.

\section{Patient consent for publication}

Not applicable.

\section{Competing interests}

The authors declare that they have no competing interests.

\section{Authors' information}

AIGO is M.D. in obstetrics-gynecology and Ph.D. student at Iuliu Hatieganu University of Medicine and Pharmacy, Cluj-Napoca, Romania. Her research topic is focused on endometriosis and the possibility of its malignant transformation. The field of research includes molecular and serological markers of this pathology. Another topic of interest is reproductive medicine.

CB is associate professor at the Research Center for Functional Genomics, Biomedicine and Translational Medicine, Iuliu Hatieganu University of Medicine and Pharmacy, Cluj-Napoca, Romania. Her main activities and responsibilities comprise functional genomics studies (miRNA and mRNA), molecular characterization and targeted therapies and genetic and genomic methods applied in molecular diagnosis.

DG is a biologist and Ph.D. student at the Research Center for Functional Genomics, Biomedicine and Translational Medicine, Iuliu Hatieganu University of Medicine and Pharmacy, Cluj-Napoca, Romania. Her main activities and responsibilities are focused on recombinant DNA technology, DNA and RNA extraction, genome editing and non-coning RNAs in cancer.

RC is M.D. in obstetrics-gynecology and Associate Professor Ph.D. at the Department of Obstetrics-Gynecology,
Iuliu Hatieganu University of Medicine and Pharmacy, Cluj-Napoca, Romania. Among his topics of interest are endometrial cancer and endometriosis, with reference to clinical and research issues.

DM is M.D. in obstetrics-gynecology, Professor Ph.D. and Chef of the Department of Obstetrics-Gynecology, Iuliu Hatieganu University of Medicine and Pharmacy, Cluj-Napoca, Romania. Among his topics of interest is endometriosis, with reference to clinical and research issues.

HR is M.D. in gynecology, surgeon of deep endometriosis at the Center of Endometriosis, Clinique Tivoli Ducos, Bordeaux, France and Professor Ph.D. at University of Aarhus, Denmark. Endometriosis represents his main topic of interest with an impressive number of publications in this field and $>100$ presentations and conferences in international and French meetings.

AI is M.D. in oncological surgery, Professor Ph.D. at the Department of Oncological Surgery, Iuliu Hatieganu University of Medicine and Pharmacy, Cluj-Napoca, Romania. Among his topics of interest is endometriosis, with reference to the possibility of malignant transformation of endometriotic lesions.

IBN is Professor Ph.D. and Director of the Research Center of Functional Genomics, Biomedicine and Translational Medicine, Director of the Research Center for Advanced Medicine MedFuture at the University of Medicine and Pharmacy, Professor of Immunology-Department of Oncology and Head of the Functional Genomics Platform for Cancer, Iuliu Hatieganu University of Medicine and Pharmacy, Cluj-Napoca, Romania. She presents an impressive number of publications, international and national projects.

\section{References}

1. Munksgaard PS and Blaakaer J: The association between endometriosis and gynecological cancers and breast cancer: A review of epidemiological data. Gynecol Oncol 123: 157-63, 2011.

2. Hsiao KY, Wu MH and Tsai SJ: Epigenetic regulation of the pathological process in endometriosis. Reprod Med Biol 16: 314-319, 2017.

3. Busacca M and Vignali M: Ovarian endometriosis: From pathogenesis to surgical treatment. Curr Opin Obstet Gynecol 15: 321-326, 2003.

4. Sampson JA: Metastatic or embolic endometriosis, due to the menstrual dissemination of endometrial tissue into the venous circulation. Am J Pathol 3: 93-110.43, 1927.

5. Jiang X, Hitchcock A, Bryan EJ, Watson RH, Englefield P, Thomas EJ and Campbell IG: Microsatellite analysis of endometriosis reveals loss of heterozygosity at candidate ovarian tumor suppressor gene loci. Cancer Res 56: 3534-3539, 1996.

6. Scarfone G, Bergamini A, Noli S, Villa A, Cipriani S, Taccagni G, Vigano' P, Candiani M, Parazzini F and Mangili G: Characteristics of clear cell ovarian cancer arising from endometriosis: A two center cohort study. Gynecol Oncol 133: 480-484, 2014.

7. Kvaskoff M, Mu F, Terry KL, Harris HR, Poole EM, Farland L and Missmer SA: Endometriosis: A high-risk population for major chronic diseases? Hum Reprod Update 21: 500-516, 2015.

8. Pearce CL, Templeman C, Rossing MA, Lee A, Near AM, Webb PM, Nagle CM, Doherty JA, Cushing-Haugen KL, Wicklund KG, et al: Association between endometriosis and risk of histological subtypes of ovarian cancer: A pooled analysis of case-control studies. Lancet Oncol 13: 385-394, 2012.

9. Lee AW, Templeman C, Stram DA, Beesley J, Tyrer J, Berchuck A, Pharoah PP, Chenevix-Trench G and Pearce CL; Ovarian Cancer Association Consortium: Evidence of a genetic link between endometriosis and ovarian cancer. Fertil Steril 105: 35-43.e1-e10, 2016. 
10. Lu Y, Cuellar-Partida G, Painter JN, Nyholt DR; Australian Ovarian Cancer Study; International Endogene Consortium (IEC), Morris AP, Fasching PA, Hein A, Burghaus S, et al: Shared genetics underlying epidemiological association between endometriosis and ovarian cancer. Hum Mol Genet 24: 5955-5964, 2015.

11. Sayasneh A, Tsivos D and Crawford R: Endometriosis and ovarian cancer: A systematic review. ISRN Obstet Gynecol 2011: 140310, 2011

12. Heidemann LN, Hartwell D, Heidemann CH and Jochumsen KM: The relation between endometriosis and ovarian cancer-a review. Acta Obstet Gynecol Scand 93: 20-31, 2014.

13. Nezhat FR, Apostol R, Nezhat C and Pejovic T: New insights in the pathophysiology of ovarian cancer and implications for screening and prevention. Am J Obstet Gynecol 213: 262-267, 2015.

14. Zafrakas M, Grimbizis G, Timologou A and Tarlatzis BC: Endometriosis and ovarian cancer risk: A systematic review of epidemiological studies. Front Surg 1: 14, 2014.

15. Sauriol A, Simeone K, Portelance L, Meunier L, Leclerc-Desaulniers K, de Ladurantaye M, Chergui M, Kendall-Dupont J, Rahimi K, Carmona E, et al: Modeling the diversity of epithelial ovarian cancer through ten novel well characterized cell lines covering multiple subtypes of the disease. Cancers (Basel) 12: 2222, 2020.

16. Webb PM and Jordan SJ: Epidemiology of epithelial ovarian cancer. Best Pract Res Clin Obstet Gynaecol 41: 3-14, 2017.

17. Gray S, Khor XY and Yiannakis D: Niraparib as maintenance therapy in a patient with ovarian cancer and brain metastases. BMJ Case Rep 12: e230738, 2019.

18. Guo SW: Endometriosis and ovarian cancer: Potential benefits and harms of screening and risk-reducing surgery. Fertil Steril 104: 813-830, 2015 .

19. Braicu OL, Budisan L, Buiga R, Jurj A, Achimas-Cadariu P, Pop LA, Braicu C, Irimie A and Berindan-Neagoe I: miRNA expression profiling in formalin-fixed paraffin-embedded endometriosis and ovarian cancer samples. Onco Targets Ther 10 4225-4238, 2017

20. Saare M, Rekker K, Laisk-Podar T, Rahmioglu N, Zondervan K Salumets A, Götte M and Peters M: Challenges in endometriosis miRNA studies-From tissue heterogeneity to disease specific miRNAs. Biochim Biophys Acta Mol Basis Dis 1863: 2282-2292, 2017.

21. Braicu C, Catana C, Calin GA and Berindan-Neagoe I: NCRNA combined therapy as future treatment option for cancer. Curr Pharm Des 20: 6565-6574, 2014.

22. Hamberg M, Backes C, Fehlmann T, Hart M, Meder B, Meese E and Keller A: MiRTargetLink-miRNAs, genes and interaction networks. Int J Mol Sci 17: 564, 2016.

23. Burney RO, Hamilton AE, Aghajanova L, Vo KC, Nezhat CN Lessey BA and Giudice LC: MicroRNA expression profiling of eutopic secretory endometrium in women with versus without endometriosis. Mol Hum Reprod 15: 625-631, 2009.

24. Forte A, Cipollaro M and Galderisi U: Genetic, epigenetic and stem cell alterations in endometriosis: New insights and potential therapeutic perspectives. Clin Sci (Lond) 126: 123-138, 2014.

25. Verma GP: Fundamentals of histology. New Delhi, New Age, 2001.

26. Cameron RI and Allen DC: Histopathology specimens clinical, pathological and laboratory aspects. London, Springer, 2005.

27. Nucci MR and Oliva E, (eds). Gynecologic pathology: Edinburgh: Churchill Livingstone, (Foundations in diagnostic pathology), pp710,2009.

28. Grandi G, Toss A, Cortesi L, Botticelli L, Volpe A and Cagnacci A: The association between endometriomas and Ovarian Cancer: Preventive effect of inhibiting ovulation and menstruation during reproductive life. Biomed Res Int 2015: 751571, 2015.

29. Kurman RJ and Shih IM: Molecular pathogenesis and extraovarian origin of epithelial ovarian cancer-shifting the paradigm. Hum Pathol 42: 918-931, 2011.

30. Kim HS, Kim TH, Chung HH and Song YS: Risk and prognosis of ovarian cancer in women with endometriosis: A meta-analysis. Br J Cancer 110: 1878-1890, 2014.

31. McCluggage WG: Morphological subtypes of ovarian carcinoma: A review with emphasis on new developments and pathogenesis. Pathology 43: 420-432, 2011

32. Worley MJ, Welch WR, Berkowitz RS and Ng SW: Endometriosis-associated ovarian cancer: A review of pathogenesis. Int J Mol Sci 14: 5367-5379, 2013.
33. Fukunaga M, Nomura K, Ishikawa E and Ushigome S: Ovarian atypical endometriosis: Its close association with malignant epithelial tumours. Histopathology 30: 249-255, 1997.

34. Ballouk F, Ross JS and Wolf BC: Ovarian endometriotic cysts. An analysis of cytologic atypia and DNA ploidy patterns. Am J Clin Pathol 102: 415-419, 1994

35. Chene G, Ouellet V, Rahimi K, Barres V, Provencher D and Mes-Masson AM: The ARID1A pathway in ovarian clear cell and endometrioid carcinoma, contiguous endometriosis, and benign endometriosis. Int J Gynaecol Obstet 130: 27-30, 2015.

36. Worley MJ Jr, Liu S, Hua Y, Kwok JS, Samuel A, Hou L, Shoni M, Lu S, Sandberg EM, Keryan A, et al: Molecular changes in endometriosis-associated ovarian clear cell carcinoma. Eur J Cancer 51: 1831-1842, 2015.

37. Pavlidou A and Vlahos NF: Endometriosis and ovarian cancer: Clinical and molecular aspects. Minerva Endocrinol 39: 155-165, 2014.

38. Matsumoto T, Yamazaki M, Takahashi H, Kajita S, Suzuki E, Tsuruta T and Saegusa M: Distinct $\beta$-catenin and PIK3CA mutation profiles in endometriosis-associated ovarian endometrioid and clear cell carcinomas. Am J Clin Pathol 144: 452-463, 2015.

39. Govatati S, Challa K, Reddy SB, Pramod K, Deenadayal M, Chakravarty B, Shivaji S and Bhanoori M: BRCA1 alterations are associated with endometriosis, but BRCA2 alterations show no detectable endometriosis risk: A study in Indian population. J Assist Reprod Genet 32: 277-285, 2015.

40. Sáinz de la Cuesta R, Izquierdo M, Cañamero M, Granizo JJ and Manzarbeitia F: Increased prevalence of p53 overexpression from typical endometriosis to atypical endometriosis and ovarian cancer associated with endometriosis. Eur J Obstet Gynecol Reprod Biol 113: 87-93, 2004.

41. Dawson A, Fernandez ML, Anglesio M, Yong PJ and Carey MS: Endometriosis and endometriosis-associated cancers: Aew insights into the molecular mechanisms of ovarian cancer development. Ecancermedicalscience 12: 803, 2018

42. Wei JJ, William $\mathbf{J}$ and Bulun S: Endometriosis and ovarian cancer: A review of clinical, pathologic, and molecular aspects. Int J Gynecol Pathol 30: 553-568, 2011.

43. Lakhani SR, Manek S, Penault-Llorca F, Flanagan A, Arnout L, Merrett S, McGuffog L, Steele D, Devilee P, Klijn JG, et al: Pathology of ovarian cancers in BRCA1 and BRCA2 carriers. Clin Cancer Res 10: 2473-2481, 2004.

44. King CM, Barbara C, Prentice A, Brenton JD and Charnock-Jones DS: Models of endometriosis and their utility in studying progression to ovarian clear cell carcinoma. J Pathol 238: 185-196, 2016

45. Bayramoğlu H and Düzcan E: Atypical epithelial changes and mutant p53 gene expression in ovarian endometriosis. Pathol Oncol Res 7: 33-38, 2001

46. Aviel-Ronen S, Soriano D, Shmuel E, Schonman R, Rosenblatt K, Zadok O, Vituri A, Seidman D, Barshack I and Cohen Y: Surgically treated ovarian endometriosis association with BRCA1 and BRCA2 mutations. Pathol Res Pract 210: 250-255, 2014.

47. Stewart CJ, Leung Y, Walsh MD, Walters RJ, Young JP and Buchanan DD: KRAS mutations in ovarian low-grade endometrioid adenocarcinoma: Association with concurrent endometriosis. Hum Pathol 43: 1177-1183, 2012.

48. Lin S and Gregory RI: MicroRNA biogenesis pathways in cancer. Nat Rev Cancer 15: 321-333, 2015.

49. Wang F, Chen C and Wang D: Circulating microRNAs in cardiovascular diseases: From biomarkers to therapeutic targets. Front Med 8: 404-418, 2014

50. Absalon S, Kochanek DM, Raghavan V and Krichevsky AM: MiR-26b, upregulated in Alzheimer's disease, activates cell cycle entry, tau-phosphorylation, and apoptosis in postmitotic neurons. J Neurosci 33: 14645-14659, 2013.

51. Miñones-Moyano E, Porta S, Escaramís G, Rabionet R, Iraola S, Kagerbauer B, Espinosa-Parrilla Y, Ferrer I, Estivill X and Martí E: MicroRNA profiling of Parkinson's disease brains identifies early downregulation of miR-34b/c which modulate mitochondrial function. Hum Mol Genet 20: 3067-3078, 2011.

52. Beuzelin D and Kaeffer B: Exosomes and miRNA-Loaded biomimetic nanovehicles, a focus on their potentials preventing type-2 diabetes linked to metabolic syndrome. Front Immunol 9: 2711,2018

53. Sung J, Wang Y, Chandrasekaran S, Witten DM and Price ND: Molecular signatures from omics data: From chaos to consensus. Biotechnol J 7: 946-957, 2012.

54. Calin GA and Croce CM: MicroRNA-cancer connection: The beginning of a new tale. Cancer Res 66: 7390-7394, 2006. 
55. Coutinho LM, Ferreira MC, Rocha ALL, Carneiro MM and Reis FM: New biomarkers in endometriosis. Adv Clin Chem 89: 59-77, 2019.

56. Ahn SH, Singh V and Tayade C: Biomarkers in endometriosis: Challenges and opportunities. Fertil Steril 107: 523-532, 2017.

57. Rekker K, Saare M, Roost AM, Kaart T, Sõritsa D, Karro H, Sõritsa A, Simón C, Salumets A and Peters M: Circulating miR-200-family micro-RNAs have altered plasma levels in patients with endometriosis and vary with blood collection time. Fertil Steril 104: 938-946.e2, 2015.

58. Agrawal S, Tapmeier T, Rahmioglu N, Kirtley S, Zondervan K and Becker C: The miRNA Mirage: How close are we to finding a non-invasive diagnostic biomarker in endometriosis? A systematic review. Int J Mol Sci 19: 599, 2018

59. Jia SZ, Yang Y, Lang J, Sun P and Leng J: Plasma miR-17-5p, miR-20a and miR-22 are down-regulated in women with endometriosis. Hum Reprod 28: 322-330, 2013.

60. Nisenblat V, Sharkey DJ, Wang Z, Evans SF, Healey M, Ohlsson Teague EMC, Print CG, Robertson SA and Hull ML: Plasma miRNAs display limited potential as diagnostic tools for endometriosis. J Clin Endocrinol Metab 104: 1999-2022, 2019.

61. Tomuleasa C, Braicu C, Irimie A, Craciun L and Berindan-Neagoe I: Nanopharmacology in translational hematology and oncology. Int J Nanomedicine 9: 3465-3479, 2014

62. Ross JS, Carlson JA and Brock G: miRNA: The new gene silencer. Am J Clin Pathol 128: 830-836, 2007.

63. Cancer Genome Atlas Research Network: Integrated genomic analyses of ovarian carcinoma. Nature 474: 609-615, 2011.

64. Zhang M, Wang S, Tang L, Wang X, Zhang T, Xia X and Fang X: Downregulated circular RNA hsa_circ_0067301 regulates epithelial-mesenchymal transition in endometriosis via the miR-141/Notch signaling pathway. Biochem Biophys Res Commun 514: 71-77, 2019.

65. Cao J, Cai J, Huang D, Han Q, Chen Y, Yang Q, Yang C, Kuang Y, Li D and Wang Z: miR-335 represents an independent prognostic marker in epithelial ovarian cancer. Am J Clin Pathol 141: 437-442, 2014

66. Liu T, Qin W, Hou L and Huang Y: MicroRNA-17 promotes normal ovarian cancer cells to cancer stem cells development via suppression of the LKB1-p53-p21/WAF1 pathway. Tumour Biol 36: 1881-1893, 2015.

67. Corney DC, Hwang CI, Matoso A, Vogt M, Flesken-Nikitin A, Godwin AK, Kamat AA, Sood AK, Ellenson LH, Hermeking H and Nikitin AY: Frequent downregulation of miR-34 family in human ovarian cancers. Clin Cancer Res 16: 1119-1128, 2010.

68. Qu W, Chen X, Wang J, Lv J and Yan D: MicroRNA-1 inhibits ovarian cancer cell proliferation and migration through c-Met pathway. Clin Chim Acta 473: 237-244, 2017.

69. Gulei D, Raduly L, Broseghini E, Ferracin $M$ and Berindan-Neagoe I: The extensive role of miR-155 in malignant and non-malignant diseases. Mol Aspects Med 70: 33-56, 2019.

70. Liu HY, Zhang YY, Zhu BL, Feng FZ, Yan H, Zhang HY and Zhou B: miR-21 regulates the proliferation and apoptosis of ovarian cancer cells through PTEN/PI3K/AKT. Eur Rev Med Pharmacol Sci 23: 4149-4155, 2019.
71. Miao C, Zhang J, Zhao K, Liang C, Xu A, Zhu J, Wang Y, Hua Y, Tian Y, Liu S, et al: The significance of microRNA-148/152 family as a prognostic factor in multiple human malignancies: A meta-analysis. Oncotarget 8: 43344-43355, 2017.

72. Xiong Y, Liu Y, Xiong W, Zhang L, Liu H, Du Y and Li N: Hypoxia-inducible factor $1 \alpha$-induced epithelial-mesenchymal transition of endometrial epithelial cells may contribute to the development of endometriosis. Hum Reprod 31: 1327-1338, 2016.

73. Du Y, Zhang Z, Xiong W, Li N, Liu H, He H, Li Q, Liu Y and Zhang L: Estradiol promotes EMT in endometriosis via MALAT1/miR200s sponge function. Reproduction 157: 179-188, 2019.

74. Ren T, Wang S, Sun J, Qu JM, Xiang Y, Shen K and Lang JH: Endometriosis is the independent prognostic factor for survival in Chinese patients with epithelial ovarian carcinoma. J Ovarian Res 10: 67, 2017

75. Barreta A, Sarian L, Ferracini AC, Eloy L, Brito ABC, de Angelo Andrade L and Derchain S: Endometriosis-associated ovarian cancer: Population characteristics and prognosis. Int J Gynecol Cancer 28: 1251-1257, 2018.

76. Park JY, Kim DY, Suh DS, Kim JH, Kim YM, Kim YT and Nam JH: Significance of ovarian endometriosis on the prognosis of ovarian clear cell carcinoma. Int J Gynecol Cancer 28: 11-18, 2018.

77. Czernobilsky B and Morris WJ: A histologic study of ovarian endometriosis with emphasis on hyperplastic and atypical changes. Obstet Gynecol 53: 318-323, 1979.

78. Ñiguez Sevilla I, Machado Linde F, Marín Sánchez MDP, Arense JJ, Torroba A, Nieto Díaz A and Sánchez Ferrer ML: Prognostic importance of atypical endometriosis with architectural hyperplasia versus cytologic atypia in endometriosis-associated ovarian cancer. J Gynecol Oncol 30: e63, 2019.

79. Wiegand KC, Shah SP, Al-Agha OM, Zhao Y, Tse K, Zeng T, Senz J, McConechy MK, Anglesio MS, Kalloger SE, et al: ARID1A mutations in endometriosis-associated ovarian carcinomas. N Engl J Med 363: 1532-1543, 2010.

80. Kuo KT, Mao TL, Jones S, Veras E, Ayhan A, Wang TL, Glas R, Slamon D, Velculescu VE, Kuman RJ and Shih IeM: Frequent activating mutations of PIK3CA in ovarian clear cell carcinoma. Am J Pathol 174: 1597-1601, 2009.

81. Sato N, Tsunoda H, Nishida M, Morishita Y, Takimoto Y, Kubo T and Noguchi M: Loss of heterozygosity on 10q23.3 and mutation of the tumor suppressor gene PTEN in benign endometrial cyst of the ovary: Possible sequence progression from benign endometrial cyst to endometrioid carcinoma and clear cell carcinoma of the ovary. Cancer Res 60: 7052-7056, 2000.

82. Pavone ME and Lyttle BM: Endometriosis and ovarian cancer: Links, risks, and challenges faced. Int J Womens Health 7: 663-672, 2015

This work is licensed under a Creative Commons Attribution-NonCommercial-NoDerivatives 4.0 International (CC BY-NC-ND 4.0) License. 